\title{
Two discrete-Euclidean operations based on the scaling transform
}

\author{
Gaëlle Largeteau-Skapin and Eric Andres \\ Laboratoire SIC, \\ Université de Poitiers, \\ BP 3017986962 Futuroscope Chasseneuil cédex, France \\ \{glargeteau, andres\}@sic.univ-poitiers.fr,
}

\begin{abstract}
In this paper we study the relationship between the Euclidean and the discrete world thru two operations based on the Euclidean scaling function: the discrete smooth scaling and the discrete based geometrical simplification.
\end{abstract}

Keywords: discrete geometry, operations, discrete scale, multi-representation modeller.

\section{Introduction}

The Euclidean and the discrete world are generally considered as antagonists. Both worlds have different properties and it is reflected in the operations. Operations might be trivial in one world and difficult to transpose in the other one. For instance, there isn't a satisfying discrete rotation that is at the same time one-to-one and commutative. Two primary properties of the Euclidean rotation. Boolean operations (intersection, union, difference) that are trivial in the discrete world become tedious to perform in the Euclidean world because of numerical errors. The goal of this paper is to show how the specificities of both worlds can be used to define operations with new interesting properties. To illustrate this we propose two operations: one in the discrete (discrete smooth scaling) and one in the Euclidean world (discrete based geometrical simplification). Each operation is partly performed in the other world with a digitization and/or an analytical reconstruction step. The digitization process allows us to move from the Euclidean world to the discrete world. The analytical reconstruction process allows us to move from the discrete to the Euclidean world.

The first operation that we are proposing is called "discrete smooth scaling". The idea behind this operation is to describe a discrete object in a smaller (finer) grid. We want to perform this operation without filtering or smoothing. The information in a discrete cell (pixel, voxel) can be a complex information that can't simply be smoothed. So far, discrete scaling didn't respect geometrical properties of the object (discrete edge slopes for instance)[3]. To solve this problem, we perform the dilation in the space best adapted: the Euclidean space. We perform an analytical reconstruction on the original image followed by a Euclidean scaling. The discretization provides us with the final "refined" image. This discrete 
smooth scaling operation possesses a remarkable property: the almost stability by inverse scale. If we make a discrete smooth scale of factor $\alpha \geq 1$ followed by a discrete smooth scale of factor $\beta=\frac{1}{\alpha}$ we obtain the original discrete object with an error bounded by a factor proportional to $\frac{1}{\alpha}$.

The second operation is a discrete based geometrical simplification operation. The operation consists, this time, starting with a Euclidean object, to digitize with a given grid size and then to reconstruct it. When we reconstruct a discrete object, the "shape complexity" (resulting vertice and edge number) depends on the size of the object. The smaller the object, the less complex the reconstructed object. It is however difficult to assure a topological consistence between the initial object and the reconstructed object. An interesting property of this operation is that the Hausdorff distance between the original object and the simplified object is bounded by a factor proportional to the grid size.

The interest of these two operations is that they each make use of the properties of the other world. The discrete operation uses the properties of the Euclidean world and the Euclidean operation those of the discrete world. These operations show how the duality between the discrete and the Euclidean world can be used at our advantage.

In section two, we introduce the basic notions used in this paper such as discrete analytical models, the principle of the analytical reconstruction method and the notations used through out the paper. In the third section we introduce the discrete discrete smooth scaling operation. In section four we present the discrete geometrical simplification operation. We conclude and propose some extensions in section five.

\section{Preliminaries}

\subsection{Basic notations in discrete geometry}

The following notations correspond to those given by Cohen and Kaufman in [6] and those given by Andres in [1]. We provide only a short recall of these notions.

A discrete (resp. Euclidean) point is an element of $\mathbb{Z}^{n}$ (resp. $\mathbb{R}^{n}$ ). A discrete (resp. Euclidean) object is a set of discrete (resp. Euclidean) points. We denote $p_{i}$ the $i$ th coordinate of a point $p$ of $\mathbb{Z}^{n}$. The voxel $\mathbb{V}(p) \subset \mathbb{R}^{n}$ of a discrete $n \mathrm{D}$ point $p$ is defined by $\mathbb{V}(p)=\left[p_{1}-\frac{1}{2}, p_{1}+\frac{1}{2}\right] \times \ldots \times\left[p_{n}-\frac{1}{2}, p_{n}+\frac{1}{2}\right]$. For a discrete object $D, \mathbb{V}(D)=\bigcup_{p \in D} \mathbb{V}(p)$

In this paper, we use the Hausdorff distance defined by:

Definition 1. Let $h$ be the direct Hausdorff distance: $A \subset \mathbb{R}^{n}, B \subset \mathbb{R}^{n}$, $h(A, B)=\max _{a \in A}\left(\min _{b \in B}\left(d_{2}(a, b)\right)\right)$, where $d_{2}$ is the Euclidean distance. The Hausdorff distance $H$ between $A$ and $B$ is $H(A, B)=\max (h(A, B), h(B, A))$

This paper is based on the relations between the Euclidean and the discrete world and the way operations can benefit from this duality. We present two operations that are based on the Euclidean scale function noted $S c$. We consider, without loss of generality, that the center of the scale function $S c$ is the origin. 


\subsection{Digitization and reconstruction}

The basic idea behind this paper is to profit from the possibility to travel between the discrete world $\mathbb{Z}^{n}$ and the Euclidean world $\mathbb{R}^{n}$. The transformation from the discrete to the Euclidean world is called digitization. The transformation from the Euclidean world to the discrete world is called reconstruction. The experiments presented in this paper have been conducted with the standard analytical model [1] (see also Fig. 1). The theoretical results are however not restricted to the standard analytical model and are also verified for a larger class of digitization schemes. Most of the digitization schemes commonly used seem actually to fit the definition that follows including the Bresenham algorithms, the supercover model, the naive digitization, the standard model, etc. Let us try to propose a characterisation of the digitization schemes that suit the purpose of this paper.

We consider digitization transforms defined by narrow offset areas. A narrow offset area $\mathbb{O}$ is defined for classes of Euclidean objects. It simply has to verify two fundamental conditions: A narrow offset area $\mathbb{O}(E) \subset \mathbb{R}^{n}$ of a Euclidean object $E$ must be narrow meaning that if $x \in \mathbb{O}(E) \cap \mathbb{Z}^{n} \Rightarrow \mathbb{V}(x) \cap E \neq \varnothing$. It simply requires that the digitization of an Euclidean object $E$ to be composed of pixels that are intersected by $E$. The second condition is a constructive condition. A narrow offset area must verify a stability property for the union: $\mathbb{O}(E \cup F)=$ $\mathbb{O}(E) \cup \mathbb{O}(F)$.

Definition 2. The digitization based on a narrow offset area is defined by:

$$
\begin{gathered}
\mathcal{D}: \mathcal{P}\left(\mathbb{R}^{n}\right) \longrightarrow \mathcal{P}\left(\mathbb{Z}^{n}\right) \\
\mathcal{D}(E)=\left\{p \in \mathbb{Z}^{n} \mid p \in \mathbb{O}(E)\right\}=\mathbb{O}(E) \cap \mathbb{Z}^{n}
\end{gathered}
$$

A good way to define a wide class of digitization tranforms is to define the offset area with a distance $d$.

$$
\mathbb{O}(E)=\left\{x \in \mathbb{R}^{n} \mid d(x, E) \leq \frac{1}{2}\right\}
$$

The best known discrete analytical model is called the supercover model $[2,4,11]$ with an offset defined by the Chebyshev distance $d_{\infty}$. The distance $d_{1}$ defines the closed naïve model and the distance $d_{2}$ defines the closed pythagorean model. All distances, of course, don't verify the narrowness property but many do. There exist also narrow offset areas that aren't defined with distances. This is the case for the Bresenham algorithms, the standard analytical model, the naive digitization, etc.

Digitization based on narrow offset areas verify, by construction, properties such as $\mathcal{D}(E \cup F)=\mathcal{D}(E) \cup \mathcal{D}(F) ; \mathcal{D}(E \cap F) \subset \mathcal{D}(E) \cap \mathcal{D}(F)$ and $F \subset G \Longrightarrow$ $\mathcal{D}(E) \subset \mathcal{D}(F)$. These properties ensure that we can build complex discrete objects out of a set of basic elements. We can, for instance, build all linear objects out of simplices.

Defining a reconstruction transform is much more difficult. If we want the reconstruction transform to make any sense we must define some properties that 

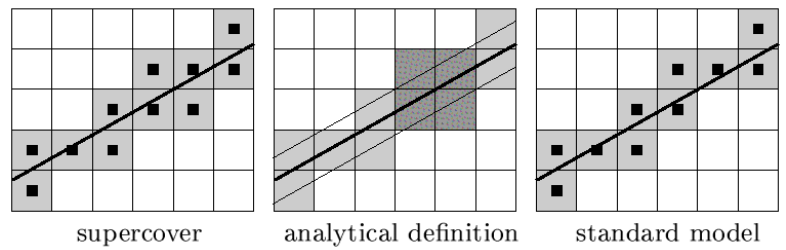

Fig. 1. supercover and standard model examples.

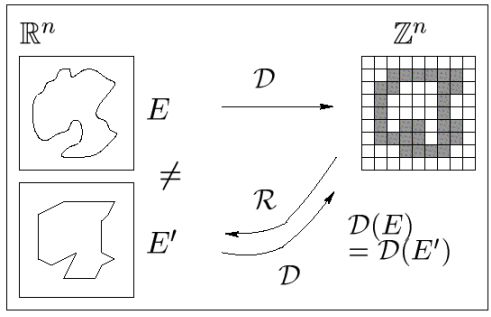

Fig. 2. Coherence between discrete and Euclidean world.

have to be verified. For any given analytical digitization, we have an infinity of reconstruction operations $[5,7,9,10]$. For instance, it's natural to associate a reconstruction transform to a digitization. Indeed, we can define a equivalence relation $\approx$ between two Euclidean objects $E$ and $F$ by $E \approx F$ iff $\mathcal{D}(E)=\mathcal{D}(F)$. There is a one-to-one mapping between the discrete objects and the equivalence classes defined by $\approx$. One of the properties of any reconstruction $\mathcal{R}$ is to stay in the equivalence class if we digitize and then reconstruct. Of course, in general, $\mathcal{R}(\mathcal{D}(E)) \neq E$ (see Fig. 2).

\section{Definition 3. Reconstruction}

A reconstruction operation $\mathcal{R}: \mathcal{P}\left(\mathbb{Z}^{n}\right) \longrightarrow \mathcal{P}\left(\mathbb{R}^{n}\right)$ associated to an analytical digitization $\mathcal{D}$ is an operation verifying, for any Euclidean object $E$ :

$$
\mathcal{R}(\mathcal{D}(E)) \approx E
$$

A property that we won't have systematically but that will be verified in many practical situations is: $\mathcal{D}(\mathcal{R}(A))=A$ for a given discrete object $A$. This property will be verified if there isn't any missing information in $A$. For instance, if we reconstruct a Bresenham line segment, that isn't missing any pixels, the property will be verified.

\section{Discrete-Euclidean operations}

In this part, we will study two operations linking the discrete and Euclidean world. The first is an operation from $\mathbb{Z}^{n}$ to $\mathbb{Z}^{n}$ that use the Euclidean scale properties to define a discrete smooth scale. The second, from $\mathbb{R}^{n}$ to $\mathbb{R}^{n}$, uses the digitization properties to erase details in Euclidean objects. 


\subsection{Discrete smooth scaling}

The first operation that we are proposing is called discrete smooth scaling. The idea behind this operation is to describe a discrete object on a smaller grid. We want to perform this operation without filtering or smoothing (see Fig. 3). We therefore perform the dilation in the space best adapted: the Euclidean space.

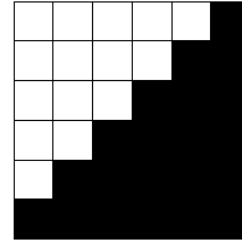

a)

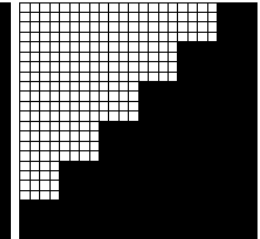

b)

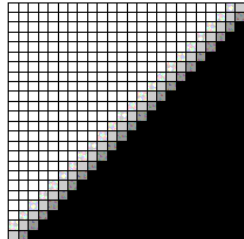

c)

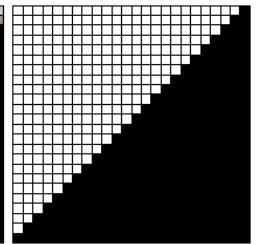

d)

Fig. 3. a) original discrete object. b)reduced grid size. c) classical smoothing. d) discrete smooth scaling.

Definition 4. We call discrete smooth scaling of a discrete object $A$ of $\mathbb{Z}^{n}$ by a scale $\alpha, \alpha \in \mathbb{R}^{+*}$, the following operation denoted $D S S_{\alpha}(A)$ :

$$
D S S_{\alpha}(A)=\mathcal{D} \circ S c_{\alpha} \circ R(A)
$$

We can see in section 4 some examples of this operation on discrete objects. The operation is meant to work for $\alpha \geq 1$. We can consider scales smaller than 1 especially in order to define the inverse operation. However the intuitive $D S S_{1}$ is actually not an exact inverse operation (see Fig. 4). We don't know for the moment how to define the exact inverse transform but we can estimate the error commited with $D S S_{\frac{1}{\alpha}}$. This error is due to the reconstruction part of the operation. We don't measure the error between two discrete objects $A$ and $D S S_{\frac{1}{\alpha}}\left(D S S_{\alpha}(A)\right)$ but between $\mathcal{R}(A)$ and $S c_{\frac{1}{\alpha}}\left(\mathcal{R}\left(D S S_{\alpha}(A)\right)\right)$. This error measure is translation independant.

Note that the error bound we are proposing makes sense for objects verifying $\mathcal{D}(\mathcal{R}(A))=A$. In case of missing information and partial information reconstruction the result of the theorem that follows stands but it's not very meaningful. Measuring an error between an incomplete discrete object and its scaled and descaled reconstruction isn't, in our case, very interesting. So, let us suppose, for what follows, that $\mathcal{D}(\mathcal{R}(A))=A$.

Let us introduce several notations: for a discrete object $A$, we note $A_{\text {first }}=$ $\mathcal{R}(A)$ the reconstruction of the original discrete object and we note $A_{\text {last }}=$ $S c_{\frac{1}{\alpha}}\left(\mathcal{R}\left(D S S_{\alpha}(A)\right)\right)$ the Euclidean object which discretization is $A_{\text {last }} \cap \mathbb{Z}^{n}=$ $D S S_{\frac{1}{\alpha}}\left(D S S_{\alpha}(A)\right)$. The error measure is a bound on the Hausdorff distance between both objects. 


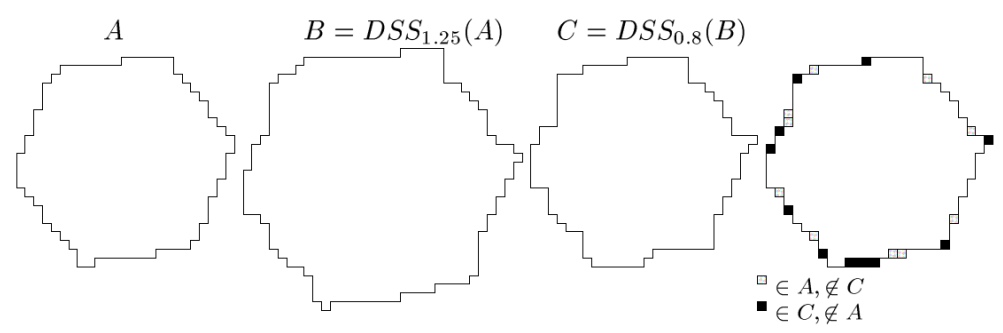

Fig. 4. Discrete smooth scaling: inversibility problem.

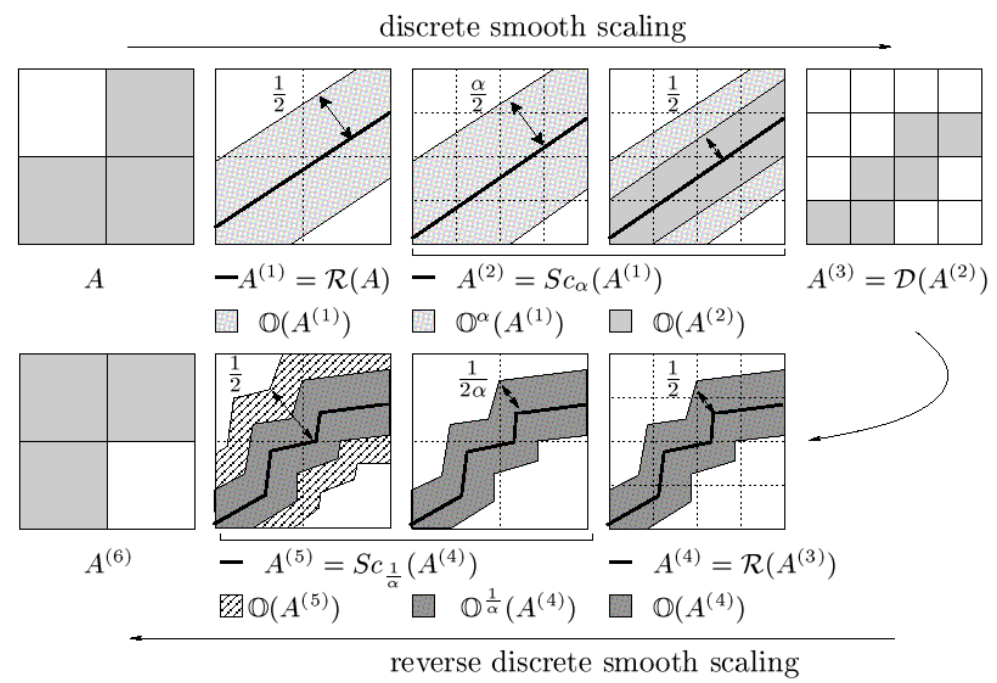

Fig. 5. Discrete smooth scaling inversibility.

\section{Theorem 1.}

$$
H\left(A_{\text {first }}, A_{\text {last }}\right)=H\left(\mathcal{R}(A), S c_{\frac{1}{\alpha}}\left(\mathcal{R}\left(\mathcal{D}\left(S c_{\alpha}(\mathcal{R}(A))\right)\right)\right)\right) \leq \frac{1}{\alpha} \sqrt{n} .
$$

Proof (see Fig. 5): Let $A$ be a discrete object and let: $A_{\text {first }}=A^{(1)}=\mathcal{R}(A) \subset$ $\mathbb{R}^{n}, A^{(2)}=S c_{\alpha}\left(A^{(1)}\right) \subset \mathbb{R}^{n}, A^{(3)}=\mathcal{D}\left(A^{(2)}\right) \subset \mathbb{Z}^{n}, A^{(4)}=\mathcal{R}\left(A^{(3)}\right) \subset \mathbb{R}^{n}$, $A_{\text {last }}=A^{(5)}=S c_{\frac{1}{\alpha}}\left(A^{(4)}\right) \subset \mathbb{R}^{n}$ and $A^{(6)}=\mathcal{D}\left(A^{(5)}\right) \subset \mathbb{Z}^{n}$. According to our notations, we have $A_{\text {first }}=A^{(1)}$ and $A_{\text {last }}=A^{(5)}$.

Digitization and reconstruction definitions and properties provide the following result: $A^{(2)} \approx A^{(4)}$ and thus $A^{(3)}=\left(\mathbb{O}\left(A^{(2)}\right)\right) \cap \mathbb{Z}^{n}=\left(\mathbb{O}\left(A^{(4)}\right)\right) \cap \mathbb{Z}^{n}$. The narrowness property of the digitization tells us that $A^{(2)}$ and $A^{(4)}$ intersect each voxel of $A^{(3)}$ and therefore, each voxel of $A^{(3)}$ contains at least one point of $A^{(2)}$ and one of $A^{(4)}$. The Euclidean distance between these two points is bounded by the voxel diagonal length: $\sqrt{n}$. We can generalize: $\forall x \in A^{(2)}, \exists y \in$ $D^{(4)} \mid d_{2}(x, y) \leq \sqrt{n}$. This implies the following result on the direct Hausdorff dis- 
tance: $\forall x \in A^{(2)}, \min _{y \in A^{(4)}}\left(d_{2}(x, y)\right) \leq \sqrt{n}$ and therefore $h\left(A^{(2)}, A^{(4)}\right) \leq \sqrt{n}$. The same reasoning stands for $h\left(A^{(4)}, A^{(2)}\right)$ and leads to $h\left(A^{(4)}, A^{(2)}\right) \leq \sqrt{n}$. The result is : $H\left(A^{(2)}, A^{(4)}\right) \leq \sqrt{n}$. We then apply the scale operation $S c_{\frac{1}{\alpha}}$. We have $H\left(S c_{\frac{1}{\alpha}}\left(A^{(2)}\right), S c_{\frac{1}{\alpha}}\left(A^{(4)}\right)\right)=H\left(A^{(1)}, A^{(5)}\right) \leq \frac{1}{\alpha} \sqrt{n}$ since $S c_{\frac{1}{\alpha}}\left(A^{(2)}\right)=A^{(1)}$ by construction and $S c_{\frac{1}{\alpha}}\left(A^{(4)}\right)=A^{(5)}$ by definition.

Finally: $H\left(A_{\text {first }}, A_{\text {last }}\right) \leq \frac{1}{\alpha} \sqrt{n}$.

Here are some comments on these results. The first obvious comment is that the bigger the scaling factor, the smaller the possible difference between $A$ and $D S S_{1}\left(D S S_{\alpha}(A)\right)$ is (in case of $\mathcal{D}(\mathcal{R}(A))=A$ as already stated). Since $\mathcal{R}(A)-$ $S c_{\frac{1}{\alpha}}\left(\mathcal{R}\left(D S S_{\alpha}(A)\right)\right)$ is smaller, there is a lesser chance that it contains a discrete point. The result of our theorem is a quite general bounding value. It doesn't take into account the fact that the reconstruction algorithms are deterministic and that it's often the case that if $A$ and $B$ are very similar then $\mathcal{R}(A)$ is similar to $\mathcal{R}(B)$. This occurs especially for small scale factors. We can thus suppose, and experimentation supports it, that in many cases the actual Hausdorff distance is much smaller than the theoretical bounding value we propose. For the case $\alpha=1$ we have no difference between $A_{\text {first }}=A_{\text {last }}$ and thus $H\left(A_{\text {first }}, A_{\text {last }}\right)=0$.

Corollary 1. $\lim _{\alpha \rightarrow \infty} H\left(A_{\text {first }}, A_{\text {last }}\right)=0$.

The corollary tells us that the discrete smooth scaling is invertible when $\alpha$ tends to infinite. In fact, the discrete smooth scaling operation can be seen as a multi-scale digitization of the Euclidean scaling function with an approximation factor $\alpha$. We can say that when $\alpha$ tends to infinite then $D S S$ tends to $S c$. Some more theoretical work needs to be done here. Non standard analysis is one way of looking at this problem [12].

\subsection{Discrete based geometrical simplification}

The second operation we have studied and implemented is a discrete based geometrical simplification operation. This operation acts on a Euclidean object that is first digitized on a given grid size and then reconstructed. According to the grid size, details are gathered in the same voxel and thus do not appear in the reconstructed object. The bigger the voxel, the lesser details from the Euclidean object will remain after the reconstruction. The object is simplified and can be represented at different levels of details (see Fig. 6). In practice, it's not the voxel size that changes but the object size. The object is scaled with the Euclidean scaling function to fit the grid size. For a scaling factor $x$ the voxel size is $\frac{1}{x}$.

Definition 5. We call discrete based geometrical simplification of a Euclidean object $E$ of $\mathbb{R}^{n}$ by a factor $\alpha, \alpha \in \mathbb{R}^{+*}$, the following operation denoted $S p_{\alpha}(E)$ :

$$
S p_{\alpha}(E)=S c_{\frac{1}{\alpha}} \circ \mathcal{R} \circ \mathcal{D} \circ S c_{\alpha}(E)
$$



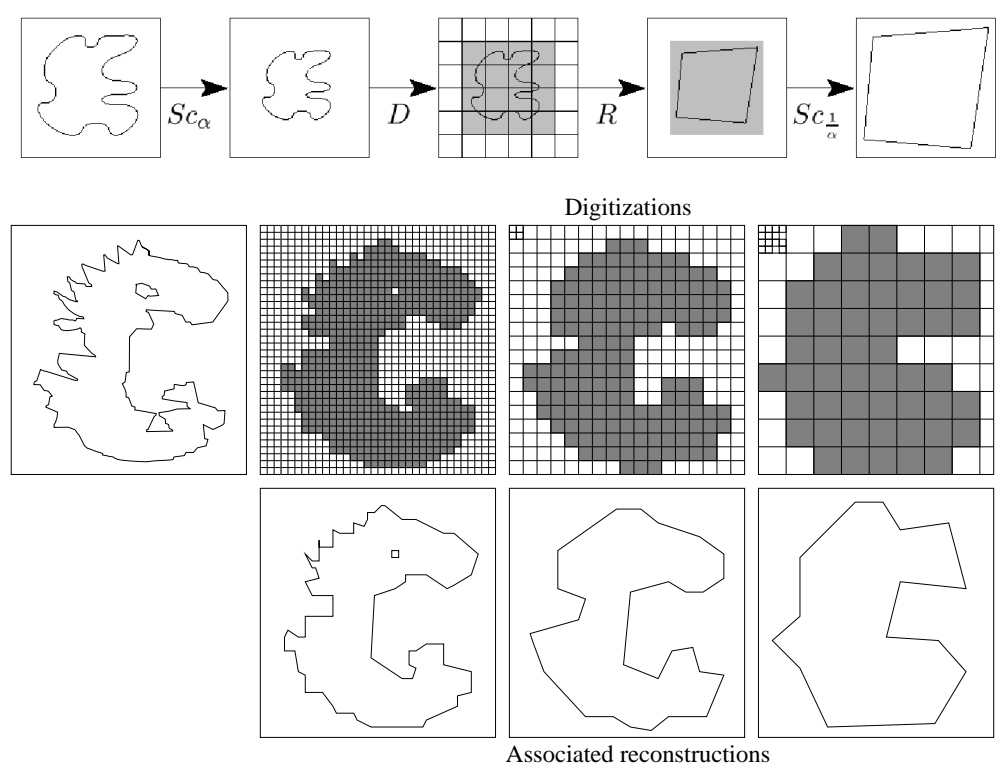

Fig. 6. Discrete based geometrical simplification principle : $\alpha=1, \frac{1}{2}$ and $\frac{1}{4}$.

We remark that the discrete based simplification has a similar property as the discrete smooth scaling operation: the Hausdorff distance between the original object and its simplification is bounded by a factor proportional to the grid size.

\section{Theorem 2.}

$$
\forall E \subset \mathbb{R}^{n}, H\left(E, S p_{\alpha}(E)\right) \leq \frac{1}{\alpha} \sqrt{n}
$$

\section{Proof:}

Theorem 2 is similar to theorem 1 . We proved that

$H\left(\mathcal{R}(A), S c_{\frac{1}{\alpha}}\left(\mathcal{R}\left(\mathcal{D}\left(S c_{\alpha}(R(A))\right)\right)\right)\right) \leq \frac{1}{\alpha} \sqrt{n}$ for $A$ a discrete object. Now, $\mathcal{R}(A)$ is a Euclidean object so, if we call $E=\mathcal{R}(A)$, we have

$H\left(E, S c_{\frac{1}{\alpha}}\left(\mathcal{R}\left(\mathcal{D}\left(S c_{\alpha}(E)\right)\right)\right)\right) \leq \frac{1}{\alpha} \sqrt{n}$. By definition $S c_{\frac{1}{\alpha}}\left(\mathcal{R}\left(\mathcal{D}\left(S c_{\alpha}(E)\right)\right)\right)=$ $S p_{\alpha}(E)$ which leads to $H\left(E, S p_{\alpha}(E)\right) \leq \frac{1}{\alpha} \sqrt{n}$.

The theorem tells us that the geometrical simplification process respects the general shape of an object. The error we commit by replacing the Euclidean object by its simplified version is bounded.

Corollary 2. $\lim _{\alpha \rightarrow \infty} S p_{\alpha}(E)=E$.

\section{Results: implementation and illustrations}

Let us comment our implementation choices and present some images to illustrate the operations. The theoretical results we presented in this paper are valid 
in dimension $n$ for a very large class of digitization and related reconstructions transforms. We implemented both operations in 2D. We present also an image of our first results in 3D.

\subsection{Implementation}

For several years our discrete geometry team develops a multi-representation modelling software intended to represent objects under four different embeddings (see Fig. 7): a Euclidean version, its analytical equivalent, the region representation and finally a discrete $2 \mathrm{D}$ pixel or $3 \mathrm{D}$ voxel representation. This allows us to choose the best adapted representation form depending on the type operation we want to realise.

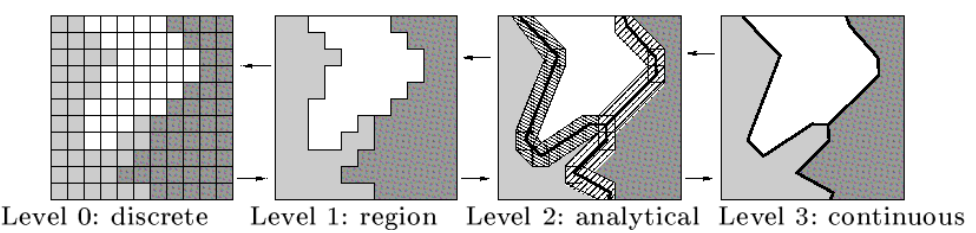

Fig. 7. Multi-representation modeller.

In this modeller discrete object are defined using the standard analytic model [1] (see Fig. 1). The reconstruction implemented in the modeller was defined in $[5,7]$ and is based on the preimage notion [8]. This algorithm computes the set of Euclidean hyperplane segments which digitization contains the original discrete object: $\mathcal{R}(A) \subset \mathbb{V}(A)$ (the standard model is a cover). This approach is based on discrete analytic geometry and is composed of two steps: the regognition of discrete analytical hyperplane segments (see [10] for an overview on recognition algorithms) and the analytical polygonalisation of the curve $[9,7]$.

\subsection{Illustrations}

Here we present illustrations of the discrete smooth scaling transform with scaling factor $\alpha=5$ and $\alpha=10$.

The reconstruction operation we implemented $[5,7]$ reconstruct objects with line segments, plane segments. The discrete smooth scaling is thus quite good on discrete objects with linear borders. The arrows in figure 8 show that on more circular parts the reconstruction creates less natural reconstruction shapes. This comes of course from the fact that a circle in low resolution will be reconstructed as a polygon.

The discrete based geometrical simplification operation decreases object detail level and therefore decreases its complexity. This operation can be used to simplify object when details are not perceptible by a human observer and when only the global pattern of the object is meaningful. Our simplification operation 


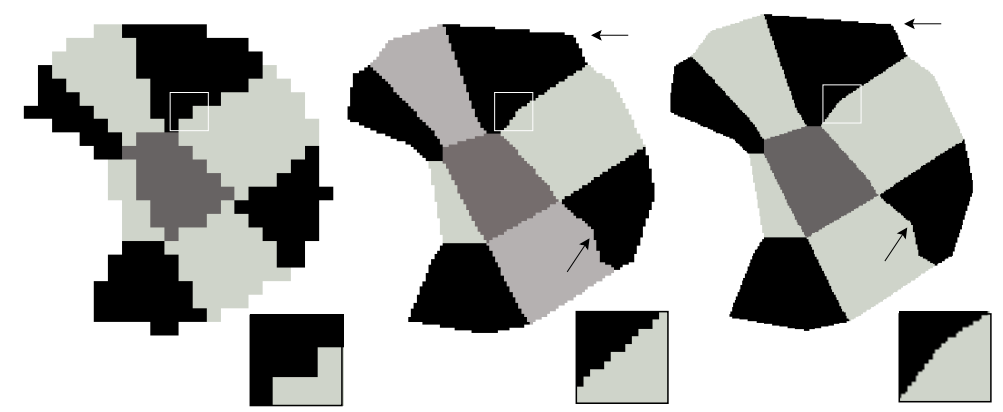

Fig. 8. Discrete smooth scale example $(\alpha=5$ and $\alpha=10)$ with details.

allows to decrease significantly the number of object element to be rendered (see Fig. 9).

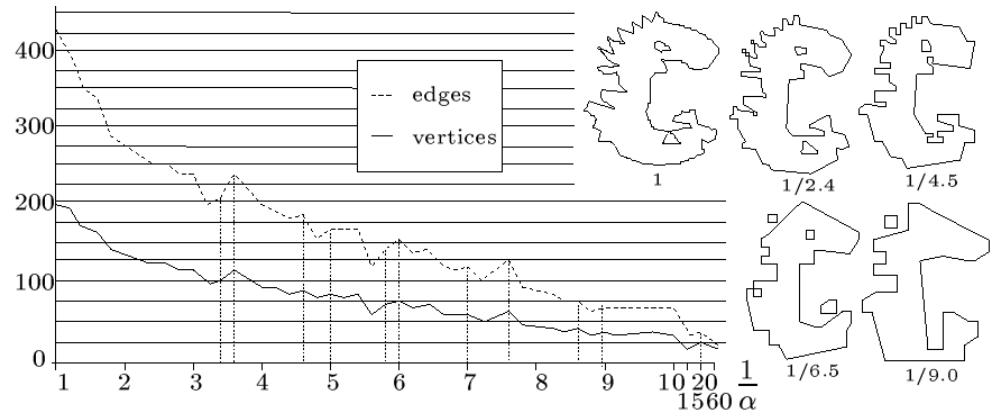

Fig. 9. Edges and vertices number evolution.

However, as we can see on figure 9, for some coefficients (see dot lines), the number of object element increases. This is due to the instability of the digitization grid resulting from a simplification with coefficients in $\mathbb{R}$ and to the non determinism of the number of reconstructed edges. Figure 9 shows several resulting pictures. We can notice that the object topology is modified: a hole can appear and then disappear. In [13], authors provide a theorem linking topology modifications and grid size. This gives only a general bound because the reconstruction process in not translation invariant. With a same grid size we can get different topologies. Object topology not only depends on the grid size but on its position. The center of the scaling function modifies the end result.

Figures 10 presents a discrete based simplification example and figure 11 shows its extention in $3 D$. 

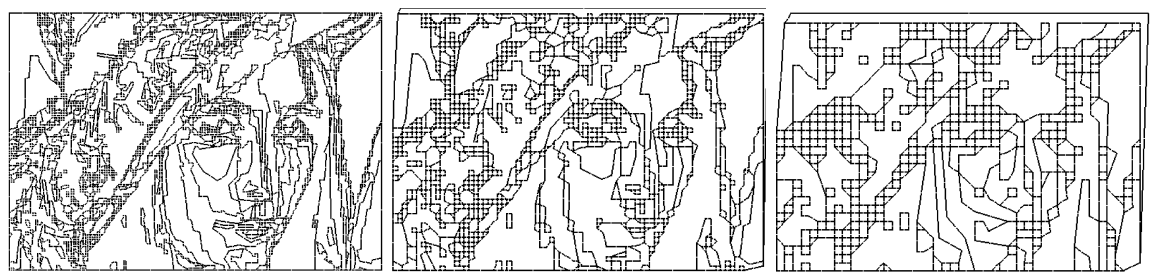

Fig. 10. Simplification example: $\alpha=1, \frac{1}{2}$, and $\frac{1}{3.5}$.

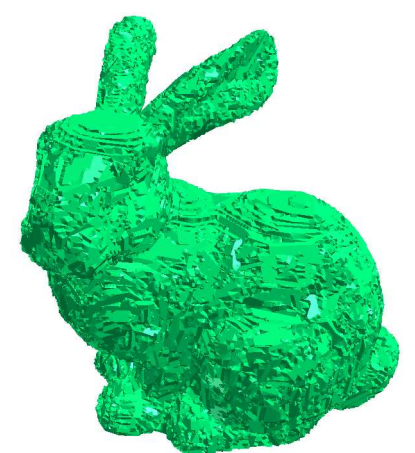

1

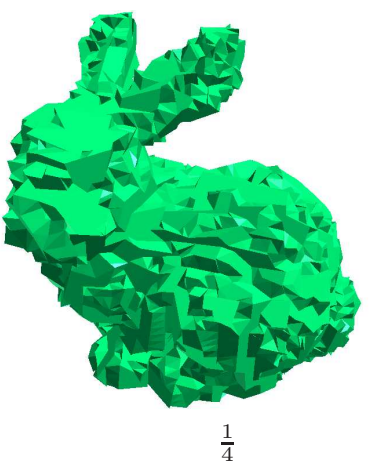

Fig. 11. $3 D$ object simplification.

\section{Conclusion}

In this paper, we have presented two operations that both use Euclidean and discrete world properties. Both operations are based on the Euclidean scaling transform. In the first case it scales the object, in the second one, it scales the grid. Both operations, while they seem quite different have a strong link and we obtained similar error bounds for them. The first operation is called the "discrete smooth scaling". We bounded the error done while trying to reverse this operation. The bigger the scale, the closer the discrete operation is to the Euclidean scaling transform. The discrete smooth scaling can be seen as a digitization of the Euclidean scale transform.

The second operation is a Euclidean operation that uses the discrete world properties. We define an operation that digitizes and recontructs Euclidean objects according to a given grid size. Depending on the grid size, a certain number of details are gathered in the same pixel and do therefore disappear during the reconstruction process. The result is a simplified Euclidean object that can be used in a multi-level representation form. The quality measure of a simplified object is a bound of the Hausdorff distance between the simplified and the original object proportional to the grid size. 
In the future we are going to consider discrete-Euclidean transforms based on Euclidean operations such as rotations, translations and general affine transforms. We are also considering discrete-Euclidean transforms based on discrete operations such as boolean operations, mathematical morphology operations, etc. The long term theoretical goal of this study is to better understand the relations between the Euclidean and the discrete world. In practice, we hope to apply this new insight in multi-level topological structure operations or on multi-scale described objects.

Acknowledgement: We would like to thank Martine Dexet for her help.

\section{References}

1. Andres,E., Menon, R., Acharya, R., Sibata, C.: Discrete linear objects in dimension $n$ : the standard model. Graphical Models, (65). Elsevier. (2003). pp. 92-111.

2. Andres, E., Acharya, R., Sibata, C.: The supercover 3D Polygon. 6th International Workshop on Discrete Geometry for Computer Imagery, Lyon, France, LNCS 1176.(1996). pp. 237-242.

3. Andres, E., Menon, R., Sibata, C., Acharya, R.: Rational bitmap scaling. Pattern Recognition Letters, Vol. 17, Number 14. (1996). pp. 1471-1475.

4. Andres, E., Nehlig, P., Franon, J.: Supercover of Straight Lines, Planes and Triangles. Discrete Geometry for Computer Imagery, 7th International Workshop, LNCS 1347.(1997). pp. 243-257.

5. Breton, R., Sivignon, I., Dupont, F., Andres, E.: Towards an invertible Euclidean reconstruction of a discrete object. Discrete Geometry for Computer Imagery, LNCS 2886, Naples. (2003). pp. 246-256.

6. Cohen-Or, D., Kaufman, A.: Fundamentals of surface voxelisation. Graph. Models Image Process.(57). (1995). pp. 453-461.

7. Dexet, M., Andres, E.: Linear discrete line recognition and reconstruction based on a generalized preimage. Int. Workshop on Combinatorial Image Analysis. Berlin. (2006). na.

8. Dorst, L., Smeulders, A.: Discrete representation of straight lines. IEEE Trans. on Pattern Anal. Mach. Intell. 6(4). (1984). pp. 450-463.

9. Françon, J., Schramm, J.M., Tajine, M.: Recognizing arithmetic straight lines and planes. 6th Int. Conf. Discrete Geometry for Computer Imagery, Springer, LNCS1176. (1996). pp. 141-150. Real Time Systems. Teknea. (2000).

10. Klette, R., Rosenfeld, A.: Digital straightness-a review. Discrete Applied Mathematics. 139(1-3). (2004). pp. 197-230.

11. Lincke, C., Wüthrich, W.: Towards a unified approach between digitization of linear objects and discrete analytical objects. in V. Skala (Ed.), Proceedings WSCG 2000 Workshop, University of West Bohemia, Plzen Tcheque Republic. (2000). pp. 124131 .

12. Reveillès, J-P.: Géométrie discrète, Calcul en nombres entiers et algorithmique. PhD thesis, Université Louis Pasteur. Strasbourg, France. (1991).

13. Tajine, M., Ronse, M.: Preservation of topolgy by Hausdorff discretization and comparison to other discretization schemes. Theoretical Computer Science, Elsevier, Vol. 283, N 1,(June 2002). pp. 243-268. 